\title{
Investigation of Mechanisms of Drug Resistance
}

National Cancer Institute

\section{Source}

National Cancer Institute. Investigation of Mechanisms of Drug Resistance. NCI

Thesaurus. Code C16038.

Basic research to understand the mechanisms that cells use to avoid being killed by cytotoxic drugs. 